\title{
Relación de los sistemas de producción con la logística en mipymes de Aguascalientes
}

Relationship of Production Systems with Logistics in MSMEs in Aguascalientes

Relação dos sistemas de produção com a logística em MPMEs em Aguascalientes

Salomón Montejano García Universidad Autónoma de Aguascalientes, México smontej@correo.uaa.mx https://orcid.org/0000-0002-4315-0768

Gabriela Citlalli López Torres Universidad Autónoma de Aguascalientes, México gabriela.lopez@edu.uaa.mx https://orcid.org/0000-0003-2483-7780

Marcelo de Jesús Pérez Ramos Universidad Autónoma de Aguascalientes, México marcelo.perez@edu.uaa.mx https://orcid.org/0000-0003-4486-3687

Rocío Montserrat Campos García Universidad Autónoma de Aguascalientes, México montserrat.campos@edu.uaa.mx https://orcid.org/0000-0003-3279-6640 


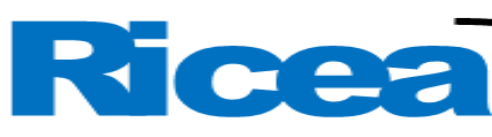

REVISTA IBEROAMERICANA DE CONTADURÍA, ECONOMÍA YADMINISTRACIÓN

ISSN= $2007-9907$

\section{Resumen}

La presente investigación busca encontrar el estado en que operan los sistemas de producción y la logística de la empresa en las micro, pequeñas y medianas empresas (mipymes) de Aguascalientes, México, así como determinar el impacto que ejercen los sistemas de producción sobre la logística de las empresas. Se trata de una investigación transversal, no experimental y cuantitativa. Para cumplir con el objetivo trazado se diseñó una encuesta que giró en torno a ambos constructos, sistemas de producción y logística empresarial, y se aplicó a 148 empresas en Aguascalientes. Para evaluar las encuestas se utilizó una escala Likert de cinco puntos. Los resultados indican que existe una correlación positiva y significativa entre ambos constructos e igualmente ponen de manifiesto que por el momento tanto los sistemas de producción como la logística de la empresa carecen de orden y de actualización en las organizaciones participantes.

Palabras clave: logística de la empresa, mipymes, sistemas de producción.

\section{Abstract}

This research seeks to find the state in which the company's production and logistics systems operate in micro, small and medium-sized enterprises (MSMEs) in Aguascalientes, Mexico. Also, it aims to determine the impact that production systems have on the logistics of companies. It is a cross-sectional, non-experimental, and quantitative investigation. To meet the objective set, a survey was designed that revolved around both constructs, production systems and business logistics, and was applied to 148 companies in Aguascalientes. A fivepoint Likert scale was used to evaluate the surveys. The results indicate that there is a positive and significant correlation between both constructs and also show that at the moment both the production systems and the logistics of the company lack order and updating in the participating organizations.

Keywords: logistics, MSMEs, production systems. 


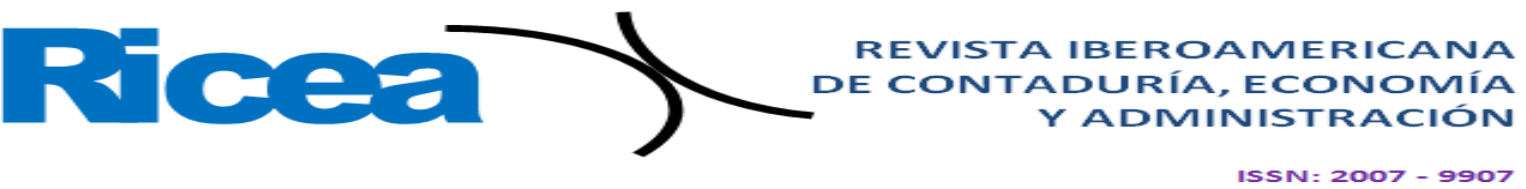

\section{Resumo}

Esta pesquisa busca conhecer o estado em que operam os sistemas produtivos e logísticos da empresa nas micro, pequenas e médias empresas (MPMEs) de Aguascalientes, México, bem como determinar o impacto que os sistemas produtivos têm na logística das empresas. É uma investigação transversal, não experimental e quantitativa. Para cumprir o objetivo traçado, foi elaborada uma pesquisa que girou em torno de construções, sistemas de produção e logística empresarial, e foi aplicada a 148 empresas em Aguascalientes. Uma escala Likert de cinco pontos foi usada para avaliar as pesquisas. Os resultados indicam que existe uma correlação positiva e significativa entre os dois construtos e também mostram que no momento tanto os sistemas de produção quanto a logística da empresa carecem de ordem e atualização nas organizações participantes.

Palavras-chave: logística empresarial, MPMEs, sistemas de produção.

Fecha Recepción: Noviembre $2020 \quad$ Fecha Aceptación: Junio 2021

\section{Introducción}

Es natural que todos los países del mundo deseen contar con una industria fuerte y poderosa para poder colocarse a la cabeza en lo referente a la fabricación y comercialización de productos y servicios a nivel mundial, sin embargo, la realidad nos muestra que países industrializados con la capacidad de dominar el mercado en realidad son muy pocos.

En 1976 nació la G7, una asociación informal conformada por siete de los países más ricos del mundo: Canadá, Francia, Alemania, Italia, Japón, Reino Unido y Estados Unidos. Se trata de un espacio todavía en activo (la última reunión se llevó a cabo en junio de 2021, después de que en 2020 fuera cancelada (Bay, 2021) para discutir estrategias y encontrar soluciones a los problemas globales siempre anteponiendo el interés de las naciones miembros. Uno de los resultados de la visión que comparte este grupo se ve reflejada en la instalación de plantas de producción u oficinas de atención en los denominados países emergentes (Milberg, 2004), los cuales, por necesidad de empleo o por dependencia tecnológica, lo aceptan. Esta situación conviene a las empresas transnacionales, derivado de los bajos costos de producción, aunado a la alta calidad del trabajo, así como la disposición de los trabajadores para laborar durante las largas jornadas de trabajo (Hymer, 1976). Independientemente de lo anterior, es muy importante tener en cuenta que toda empresa, por 


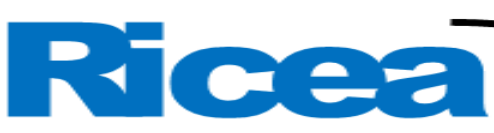

REVISTA IBEROAMERICANA DE CONTADURÍA, ECONOMÍA YADMINISTRACIÓN

ISSN= $2007-9907$

más grande que sea, incluyendo por supuesto a las transnacionales, en un determinado momento, también fueron micro, pequeñas y medianas empresas (mipymes), y que fue gracias al desarrollo del trabajo organizado y a perdurar a lo largo del tiempo que se convirtieron en las poderosas entidades que son actualmente. Igualmente, no está de más señalar que no es el tamaño de las empresas lo que refleja su poderío, sino el impacto de su presencia primeramente en sus propios países, y posteriormente en el mundo globalizado en el que estamos.

En México, las mipymes conforman prácticamente la totalidad de las empresas $(99.8 \%)$ y generan $85.6 \%$ de los empleos; sin embargo, en lo que respecta a remuneraciones e ingresos, las cifras no son tan impresionantes como las anteriores: $69.3 \%$ y $59.3 \%$, respectivamente (Instituto Nacional de Estadística y Geografía (INEGI, 2009). Lo anterior se debe a que la aportación de las mypimes más pequeñas no concuerda con el gran número de estas que existen en México, por lo que la brecha entre las más pequeñas y las más grandes empresas es cada vez mayor, lo cual se puede comprobar con tan solo observar los ingresos de estas últimas, las cuales, a pesar de ser considerablemente menos, registran cerca de la mitad de los ingresos en este país (40.7 \%) (INEGI, 2009).

Por supuesto, más que el tamaño, lo que más influye es la manera en que se administran los negocios. En efecto, a partir de los finales del siglo XIX la administración de la empresa comenzó a evolucionar y adquirir importancia hasta el punto de hacerse, en este momento, indispensable a causa de la globalización del comercio a nivel mundial, por lo que la internacionalización de las empresas es vital en todos los aspectos, incluyendo su desarrollo y actualización.

Llegados a este punto cabe señalar que las situaciones que giran alrededor del desarrollo de las mipymes no responden a una sino a múltiples variantes. Prácticamente, cualquier situación que se presenta en torno a la empresa, o al interior de esta, puede impactar considerablemente (Ruzzier, Hisrich y Antoncic, 2006). Y sin dejar de tomar en cuenta lo anterior, aquí las situaciones que pretendemos considerar prioritariamente son los sistemas de producción y la logística de la empresa. 


\section{Sistemas de producción}

A causa del compromiso inherente a la práctica empresarial, por la propia naturaleza de cubrir las necesidades de la población, que cada vez son mayores, los sistemas de producción deben estar de manera constante en renovación. La necesidad de fabricar productos para hacer la vida del ser humano lo más placentera posible siempre ha existido. Sin embargo, posiblemente dicho sentimiento se exacerbó durante el desarrollo tecnológico del siglo XIX, cuando se catapultó el desarrollo industrial.

Anteriormente, las necesidades manifestadas por la población eran diferentes en cantidad, variedad y calidad de los productos, puesto que la cantidad de personas en el mundo era aproximadamente la octava parte de lo que somos en la actualidad. Ahora bien, al incrementar las necesidades, fue necesario cambiar la manera de producir y fue a partir de la capacidad del ser humano para manejar la energía transportada por el vapor que los sistemas de producción se pudieron mecanizar para lograr cubrir las necesidades existentes en la sociedad en ese momento (Noori y Radford, 1997). Por supuesto que los sistemas de producción eran incipientes y la preocupación principal de los administradores era producir lo más posible, por lo que, sin saberlo, sacrificaban calidad, variedad, personal, medio ambiente, entre otras cosas, que por la falta de control no entendían que eran factores que les restaban competitividad.

Una vez transcurrido el tiempo, los administradores se dieron cuenta de que era posible producir aún más de lo que en ese momento se hacía al mejorar en un principio los métodos de trabajo, y se inició la transformación en este sentido (Peters, 1987). Estos cambios eran direccionados específicamente hacia las formas en que se producía, sin poner atención a otros aspectos como el humano, social y otros que en ese momento se consideraban no esenciales. Como sea que fuere, dichos esquemas de trabajo dieron resultados positivos, y fueron la base para lo que actualmente se consideran como formas modernas de trabajo.

Un avance enorme lo constituyó la producción en serie. Una vez que, basados en el principio de la especialidad del trabajo, así como la división de este, se habilitó a la empresa para producir grandes volúmenes de producción, se tuvo que actualizar la maquinaria, las instalaciones y las habilidades del personal para crear estaciones de trabajo conectadas entre sí, con la condición de que el proceso no se interrumpiera (Gershwin y Schor, 2000). Las empresas poco a poco se sumaron a esta forma de trabajo y, gracias a ello, fueron creciendo. 


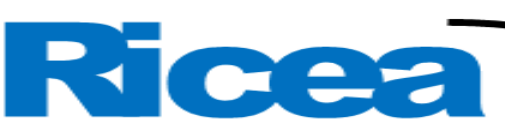

REVISTA IBEROAMERICANA DE CONTADURÍA, ECONOMIIA YADMINISTRACIÓN

ISSN $=2007-9907$

No obstante, el tiempo también alcanzó a esta forma de producción. Aunque se podía cumplir con la cantidad de producción solicitada, se tenía la limitante de la entrega en tiempo a una demanda originada por emergencias, o por la necesidad en el mercado de una variedad amplia de productos, pues los cambios de modelo para cubrir este tipo de demandas eran muy tardados, todo lo cual obligó a tener una cantidad de inventario terminado muy alta para cumplir con estas contingencias y no perder clientes, puesto que las corridas de producción no se podían interrumpir hasta que el lote programado estuviera terminado.

Derivado de lo anterior, se establecieron los sistemas de producción flexible. Dicha metodología permitió producir lotes pequeños de producción de una amplia gama de productos (Lee, 1998). Claro que para ello fue indispensable el desarrollo de equipo y maquinaria que fuera no solo capaz de realizar varias operaciones de manera sencilla, sino que también permitiera cambiar de una operación a otra de manera rápida. Se trata de maquinaria y equipo computarizado que, organizado linealmente, forma lo que se conoce como manufactura por computadora, la cual inicia en aplicaciones para moldeo por inyección, formado de metales, así como manufactura y ensamblado de piezas (Ranta y Tchijov, 1990). Sin embargo, realizar el cambio de una manera de producir a otra requería de grandes inversiones y de un alto grado de conocimiento sobre sistemas computarizados (Smith y Stecke, 1996), lo que se convirtió en una barrera para muchas empresas.

Naturalmente, la evolución de los sistemas de producción tendió hacia la automatización. Cada vez fue más evidente la necesidad de mayor control y seguridad en los procesos y la respuesta se encontró en la creación de mecanismos inteligentes, cuya operación fuera posible incluso sin operario (Ahmad, Tahir, Ul y Shah, 2019). Una vez alcanzados estos niveles de automatización, se logró mayor precisión y mayor velocidad en la producción. El resultado de esto ha permitido que se puedan manejar con determinación los cambios profundos y necesarios en los procesos industriales que se requieren en la actualidad, así como la optimización de estos (Hu, Starr y Leung, 1999)

Por otro lado, a partir de 1924 el impacto que el cuidado de la calidad tiene sobre los sistemas de producción comienza a ser relevante (Lovitt, 1997). Por efecto de la presión del cliente sobre la oferta de la empresa, los sistemas de calidad tomaron preponderancia dentro de los sistemas de trabajo con el propósito de ordenar y mejorar los resultados finales de los procesos de producción. Como consecuencia natural de la presión que se ejerció en las décadas de los 70 y 80 sobre compañías europeas y estadounidenses, los empresarios se 


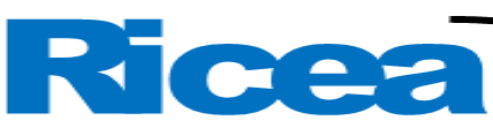

REVISTA IBEROAMERICANA DE CONTADURÍA, ECONOMÍA YADMINISTRACIÓN

ISSN : $2007-9907$

vieron en la imperiosa necesidad de tomar en serio la calidad de los productos. Paralelamente, motivadas por esta corriente, se diseñaron nuevas metodologías de trabajo, por ejemplo, seis sigma y lean manufacturing (Dahlgaard, 2011), metodologías que, en sí mismas, tienen la característica principal de mejorar sustancialmente los resultados de la organización, lo cual hace que se aconseje la inversión en estos campos (Boulter, Bendell y Dahlgaard, 2013).

\section{Logística de la empresa}

La manera de hacer negocios ha evolucionado a tal grado que ha forzado a las organizaciones a tener contacto con el resto del mundo y a sufrir o a aprovechar cualquier desbalance. Sin duda lo anterior ha impulsado el desarrollo integral de las empresas. Por ejemplo, un efecto inmediato de esta situación es la desincorporación de los procesos productivos en diferentes áreas administrativas, de acuerdo con las funciones comunes que se lleven a cabo durante las operaciones de la organización. Asimismo, ha obligado a las organizaciones a la elaboración de partes en diferentes países (Milberg, 2004). Consecuentemente, el control del proceso se ha complicado, por lo que se requiere contar con la especialización de las funciones para lograr maximizar los resultados. De tal forma que el manejo de la logística se ha convertido en una de las prioridades empresariales, imprescindible hoy en día para incrementar la capacidad de realizar de negocios, ya que estos se deben de realizar inclusive en el extranjero. Así pues, más que actuar conforme a los avances tecnológicos y por el impulso que se genera por lo realizado en empresas internacionales, es necesario que se tome en serio la efectividad de los costos, la eficiencia de las nuevas formas de trabajar y la rapidez con la que se atiende al cliente (Gola y Konczal, 2013).

Ahora bien, para muchos los términos administración de la cadena de valor y administración de la cadena de suministro pueden ser indistintos, sin embargo, aquí se conciben diferentes. Todavía más: por la evolución de los sistemas de producción se tiene la necesidad de desintegrarlos funcionalmente para apoyar directamente a la cadena de valor.

La cadena de valor se define como la secuencia de las actividades involucradas en la realización de un bien o un servicio (Noori y Radford, 1997), actividades siempre relacionadas con el cliente: desde la detección de sus necesidades hasta la evaluación de la satisfacción de estas. 


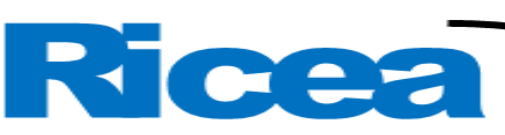

REVISTA IBEROAMERICANA DE CONTADURÍA, ECONOMIIA YADMINISTRACIÓN

ISSN $=2007-9907$

Una de las áreas que se han organizado a partir de los años 60 para funcionar de manera independiente es precisamente la que tiene que ver con la logística de la empresa (Gustaffson, 2006). En este concepto se integra lo referente a el servicio al cliente, tráfico y medios de transporte, almacenaje, selección de las instalaciones, el control de los inventarios, control de los pedidos, la realización de las compras, el movimiento de los materiales, así como la incorporación de componentes, entre otras, con el propósito de hacer llegar al cliente los productos requeridos bajo estándares de calidad, tiempo y cantidad (Jellouli y Adbelkadhi, 2013; Pascual y Ribas, 2015).

La logística de la empresa cuenta con varias definiciones. Sin embargo, de manera general podríamos definirla como la gestión estratégica de la adquisición, traslado y almacenaje de materiales y productos acabados y la información relacionada, mediante el diseño y control de los canales de distribución, con el objetivo esencial de optimizar el resultado de las operaciones de la compañía tanto en el presente como en el futuro (Bowersox, Closs, Cooper y Bowersox, 2013)

Aunque las funciones logísticas de la empresa se han especializado y desintegrado de la operación de la planta, es indispensable tener siempre presente que su objetivo primordial es el de servir directamente a los procesos de producción: poner al alcance de quien lo requiera cualquier material de entre los que se definieron cuando se realizó la lista de materiales de producción, proveer al área de manufactura de estos en el momento programado y controlar el libre flujo de la producción en proceso, tomando en consideración el cuidado y la calidad necesarios para cumplir con los requerimientos del cliente (Samaranayake y Toncich, 2007). Asimismo, la logística de la empresa está muy relacionada con el flujo de información proporcionado por los sistemas con que se cuenta en el área de operación. De igual manera, está vinculada con el estado, cuidado y capacidad de operación que tienen el equipo y la maquinaria con que se cuenta para producir. Algo que también influye en grado sumo es el estado de las instalaciones y la distribución que se tiene de los equipos, entre otras tareas que le corresponden realizar directamente al área de operación (Sandberg, 2013), y sin las cuales se afecta el desempeño del área de logística de la empresa.

Tomando en cuenta todo lo anterior, en la figura 1 se presenta el modelo que guio la presente investigación, en donde se indican las variables que se consideraron para evaluar el estado de control que tienen las mipymes de Aguascalientes, México, sobre los sistemas de producción y la logística de la empresa. 
Figura 1. Modelo de investigación que relaciona los sistemas de producción con la logística de la empresa

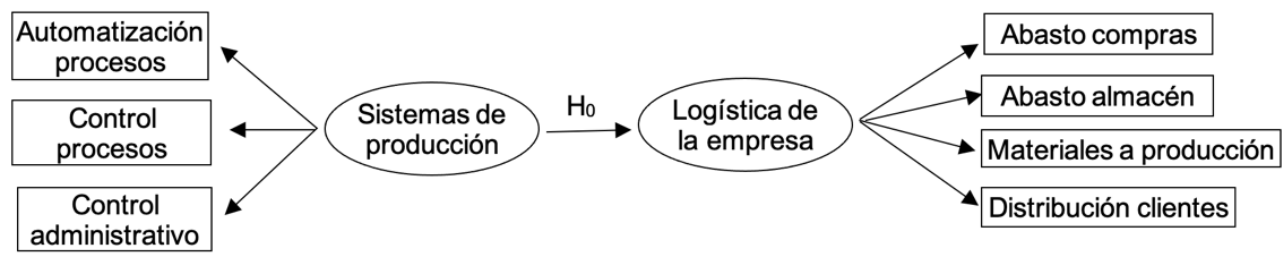

Fuente: Elaboración propia

signnicallvanlente en la iugistica ue ra emipresa.

\section{Metodología}

Esta investigación se realizó para determinar el estado que guardan los sistemas de producción y el manejo de la logística de la empresa, así como el impacto de los sistemas de producción sobre la logística de la empresa en las mipymes de Aguascalientes, México. Esta investigación es de tipo transversal, cuantitativa, no experimental. Para ello, se estructuró una encuesta para analizar el constructo Sistemas de Producción (SP), el cual se compone de tres dimensiones que analizan el estado general de dichos procesos en la empresa: 1) automatización de los procesos (con siete preguntas), 2) confiabilidad de los procesos (con tres preguntas) y 3) control administrativo de los procesos (cinco preguntas). Por otro lado, el constructo Logística Empresarial (LE) cuenta con cuatro dimensiones: 1) abasto compras (con seis preguntas), 2) abasto de almacén (con nueve preguntas), 3) materiales de producción (con siete preguntas) y 4) distribución de clientes (11 preguntas). La encuesta se aplicó en 148 empresas en Aguascalientes para determinar cómo el empresario de este estado visualiza ambos constructos en su empresa. Cabe señalar que, de las 148 empresas, $81.1 \%$ eran micros, $15.5 \%$ eran pequeñas y $3.4 \%$ medianas. 
bajo estas condiciones, se considera que se cuenta con la pertinencia necesaria para interpretar las respuestas de la encuesta como indicadoras de lo que sucede en las empresas analizadas.

Tabla 2. Valores del alfa de Cronbach y media de respuesta para los constructos analizados y sus dimensiones

\begin{tabular}{|l|l|l|}
\hline & Alfa de Cronbach & Valor medio \\
\hline Abasto compras & 0.669 & 3.2556 \\
\hline Abasto almacén & 0.805 & 3.0509 \\
\hline Materiales a producción & 0.883 & 2.8137 \\
\hline Distribución clientes & 0.871 & 2.8624 \\
\hline Control logístico & 0.694 & 2.9957 \\
\hline Automatización de procesos & 0.874 & 1.5743 \\
\hline Control del proceso & 0.861 & 3.4505 \\
\hline Control administrativo & 0.777 & 2.6463 \\
\hline Sistemas de producción & 0.659 & 2.5770 \\
\hline
\end{tabular}

Fuente: Elaboración propia

\section{Resultados}

El resultado original del estudio indica el grado de atención que el empresario le aporta a la operación de sus procesos. Idealmente hubiéramos querido que los resultados fueran excelentes en todos los aspectos, es decir, con nivel cinco; no obstante, aunque se cuenta con niveles bajos de atención, se observa que la utilización de técnicas logísticas de alguna forma es homogénea, por lo tanto, la mejora aplicada se debe realizar a todos los aspectos que tienen que ver con la logística empresarial. 


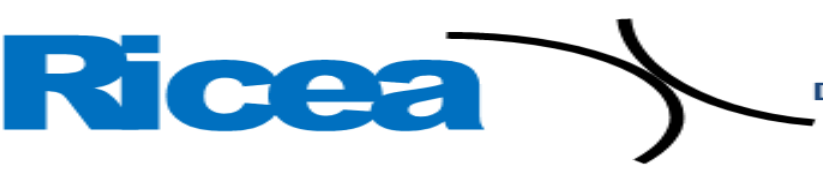

REVISTA IBEROAMERICANA DE CONTADURÍA, ECONOMIIA YADMINISTRACIÓN

Figura 2. Respuesta media para el constructo LE y sus dimensiones

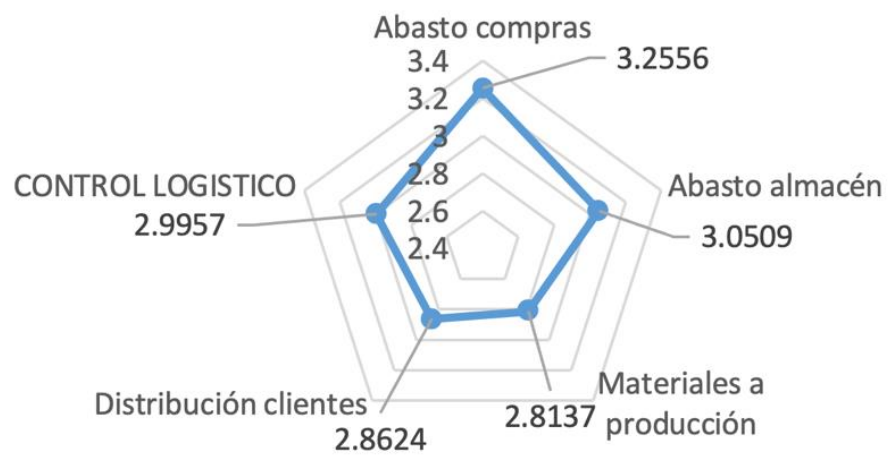

Fuente: Elaboración propia

La figura número 2 muestra que para el constructo LE el valor medio es de 2.9957, lo cual indica que en Aguascalientes se utilizan solamente algunas técnicas de control logístico de la empresa. Asimismo, indica que no se hace de manera formal y constante. En este sentido, la información presentada en la tabla 3 manifiesta que $74.3 \%$ de los empresarios reconoce que emplea en pocas ocasiones y solamente algunas técnicas conocidas de logística empresarial, en tanto que solamente $25.7 \%$ menciona que utiliza de manera constante este tipo de técnicas. 


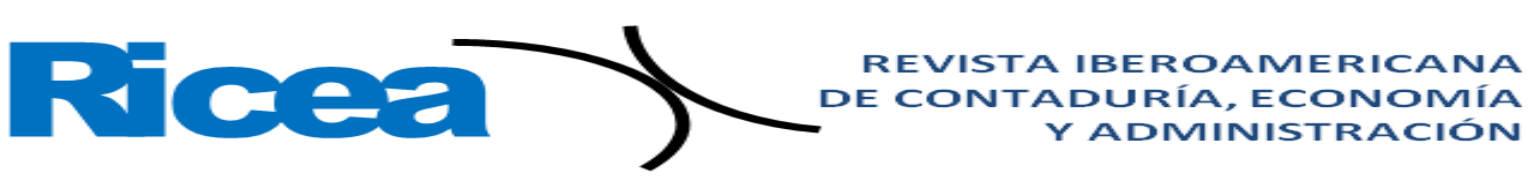

ISSN $=2007-9907$

Tabla 3. Resumen de porcentajes sencillos y acumulados de la frecuencia de respuesta para el constructo LE y sus dimensiones

\begin{tabular}{|c|c|c|c|c|c|c|c|c|c|c|c|c|}
\hline $\begin{array}{l}\text { Variables para } \\
\text { calificar el } \\
\text { constructo } \\
\text { calidad }\end{array}$ & $\begin{array}{l}\text { Respuesta } \\
\text { principal }\end{array}$ & 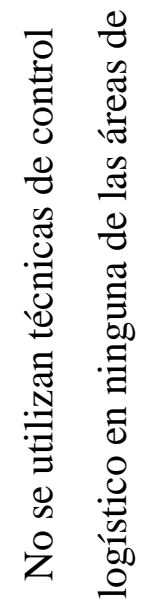 & 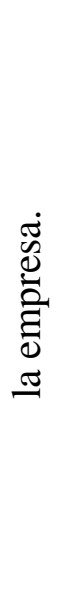 & 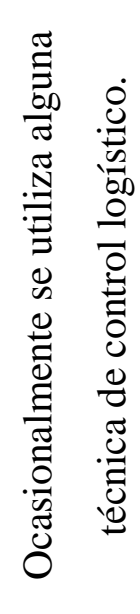 & 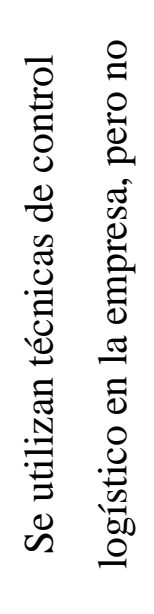 & 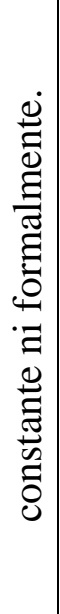 & 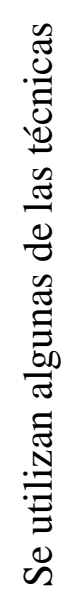 & 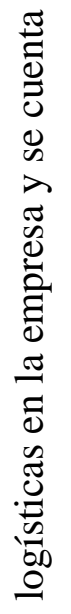 & 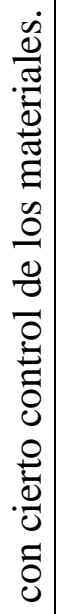 & 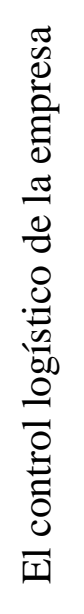 & 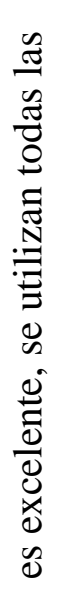 & 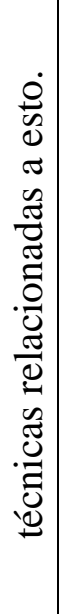 \\
\hline \multirow{3}{*}{$\begin{array}{l}\text { Abasto a } \\
\text { compras }\end{array}$} & Frecuencia & \multicolumn{2}{|l|}{4} & 25 & \multicolumn{2}{|l|}{59} & \multicolumn{3}{|l|}{43} & \multicolumn{3}{|l|}{17} \\
\hline & Porcentaje & \multicolumn{2}{|l|}{2.7} & 16.9 & \multicolumn{2}{|l|}{39.9} & \multicolumn{3}{|c|}{29.0} & \multicolumn{3}{|c|}{11.5} \\
\hline & Acumulado & \multicolumn{2}{|l|}{2.7} & 19.6 & \multicolumn{2}{|l|}{59.5} & \multicolumn{3}{|c|}{88.5} & \multicolumn{3}{|l|}{100} \\
\hline \multirow{3}{*}{ Abasto almacén } & Frecuencia & \multicolumn{2}{|l|}{9} & 32 & \multicolumn{2}{|l|}{59} & \multicolumn{3}{|l|}{31} & \multicolumn{3}{|l|}{17} \\
\hline & Porcentaje & \multicolumn{2}{|l|}{6.1} & 21.6 & \multicolumn{2}{|l|}{39.9} & \multicolumn{3}{|c|}{20.9} & \multicolumn{3}{|c|}{11.5} \\
\hline & Acumulado & \multicolumn{2}{|l|}{6.1} & 27.7 & \multicolumn{2}{|l|}{67.6} & \multicolumn{3}{|c|}{88.5} & \multicolumn{3}{|l|}{100} \\
\hline \multirow{3}{*}{$\begin{array}{l}\text { Materiales a } \\
\text { producción }\end{array}$} & Frecuencia & \multicolumn{2}{|l|}{29} & 28 & 38 & & 40 & & & 13 & & \\
\hline & Porcentaje & 19.6 & & 18.9 & 25.7 & & 27 & & & 8.8 & & \\
\hline & Acumulado & 19.6 & & 38.5 & 64.2 & & 91.2 & & & 100 & & \\
\hline & Frecuencia & 19 & & 25 & 65 & & 34 & & & 5 & & \\
\hline clientes & Porcentaje & 12.8 & & 16.9 & 43.9 & & 23.0 & & & 3.4 & & \\
\hline & Acumulado & 12.8 & & 29.7 & 73.6 & & 96.6 & & & 100 & & \\
\hline & Frecuencia & 4 & & 37 & 69 & & 31 & & & 7 & & \\
\hline & Porcentaje & 2.7 & & 25.0 & 46.6 & & 21.0 & & & 4.7 & & \\
\hline & Acumulado & 2.7 & & 27.7 & 74.3 & & 95.3 & & & 100 & & \\
\hline
\end{tabular}

Fuente: Elaboración propia

De acuerdo con la figura 2, para "Abasto a compras" el valor medio de respuesta es 3.2556, lo cual, si bien lo coloca como la dimensión mejor evaluada por los empresarios, evidencia que el empleo de técnicas enfocadas al control del abasto a compras es muy 


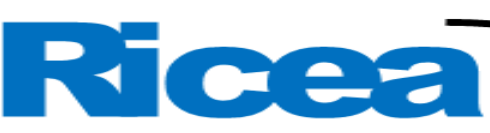

REVISTA IBEROAMERICANA DE CONTADURÍA, ECONOMIIA YADMINISTRACIÓN

ISSN $=2007-9907$

limitado y que este se lleva de manera práctica, por lo cual las oportunidades de mejora son muchas. Como complemento de esta información, en la tabla 3 se tiene que $59.5 \%$ de los empresarios menciona que, efectivamente, la aplicación de técnicas logísticas encaminadas al abasto a compras es muy limitada, en tanto que únicamente $40.5 \%$ expresa que de manera constante utilizan la mayoría de las técnicas de control para el abasto a almacenes.

Ahora bien, para la dimensión "Abasto almacén" en la figura 2 se muestra que el valor medio de respuesta es de 3.0509, lo cual expresa que en lo referente a esta dimensión la aplicación de técnicas de control es muy limitada, en otras palabras, que su aplicación es muy limitada, ya que se utilizan, pero sin un orden y una constancia adecuados. Además, en la tabla 3 se muestra que $67.6 \%$ de los encuestados afirma utilizar en pocas ocasiones y de manera superficial técnicas de control para el abasto de almacén; únicamente $32.4 \%$ de las empresas utilizan estas técnicas regularmente y en una variedad actualizada.

En lo concerniente a lo que son los materiales a producción, en la figura 2 es posible observar que el valor medio de respuesta es de 2.8137. Se trata de la dimensión con menor calificación: son muy pocas las técnicas de logística que se utilizan y que se aplican; solamente se hace en contadas ocasiones o con algunos procesos, pero la mayoría se encuentran fuera de control. En la tabla 3, por su parte, se muestra que $64.2 \%$ de las empresas aplica muy poco las técnicas relacionadas con el control y el suministro de los materiales a producción, así como el retorno del producto al almacén, por lo que esto se convierte en un punto de caos durante la etapa de producción. De igual forma, se puede ver que únicamente $35.8 \%$ de las empresas utiliza las técnicas de control de los materiales a producción de manera regular y constante durante sus operaciones.

Por último, para la dimensión "Distribución clientes", la figura 2 deja ver que el valor medio de respuesta de los empresarios en Aguascalientes es de 2.8624, lo cual apunta a que la utilización de técnicas relacionadas con la distribución a clientes es muy reducida y únicamente en algunas ocasiones se pone atención a esta parte de la cadena de suministro. Igualmente, en la tabla 3 se observa que $74.3 \%$ de los empresarios responde que no utilizan técnicas de control distribución clientes y quienes lo hacen es de manera esporádica y con pocas técnicas aplicadas; $25.7 \%$ expresa que regularmente utiliza estas técnicas. 
Figura 3. Respuesta media para SP y sus dimensiones

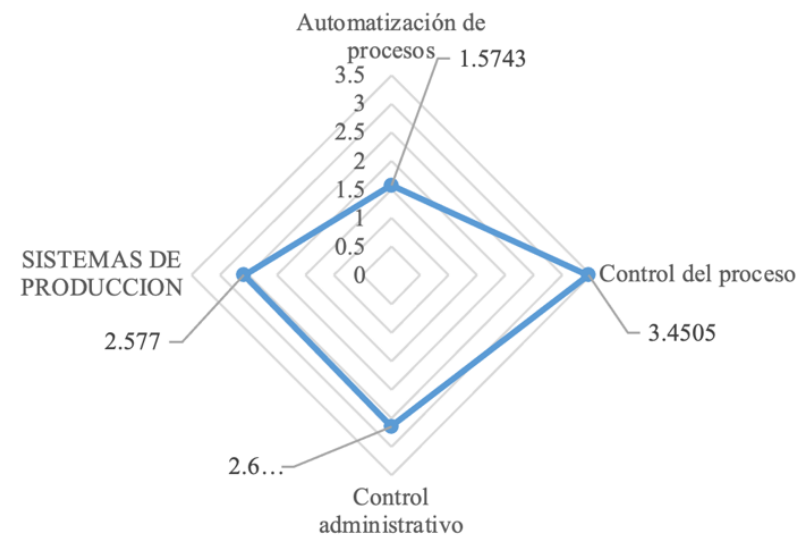

Fuente: Elaboración propia

Para apoyar en el análisis del constructo SP y sus dimensiones, los resultados se graficaron tal y como se muestran en la figura 3. En esta podemos observar que, además de ser reducido el control que se tiene sobre el constructo en cuestión, los resultados en las dimensiones son muy heterogéneos.

En cuanto al control sobre los sistemas de producción, la figura 3 revela una respuesta media de 2.577, lo cual indica que solamente algunas de las técnicas de control en los sistemas de producción, las más conocidas, son empleadas de manera empírica por los empresarios, por lo que se manifiesta una falta de actualización técnica de estas empresas en Aguascalientes. Asimismo, en la tabla 4 se observa que $87.2 \%$ de los empresarios expresa utilizar pocas técnicas de control y que lo hacen sin formalidad y esporádicamente, y únicamente $12.8 \%$ reconoce que tiene un control adecuado de sus sistemas de producción y que de manera constante se hacen esfuerzos para mantenerse al día en este rubro.

La dimensión "Automatización de los procesos" cuenta con una respuesta media de 1.5743, la menor calificación registrada, y por lo tanto, la más descuidada del constructo SP. Este resultado quiere decir que las mipymes en Aguascalientes no cuenta en grado alguno con procesos automatizados, situación que se confirma en lo expuesto en la tabla 4, que expresa que $96.6 \%$ de los empresarios expresa que las técnicas de control en los sistemas de producción son prácticamente empíricos y que ocasionalmente se trabaja con algún sistema automático (solamente 3.4 \% expresó la aplicación de técnicas de control en los sistemas de producción). 


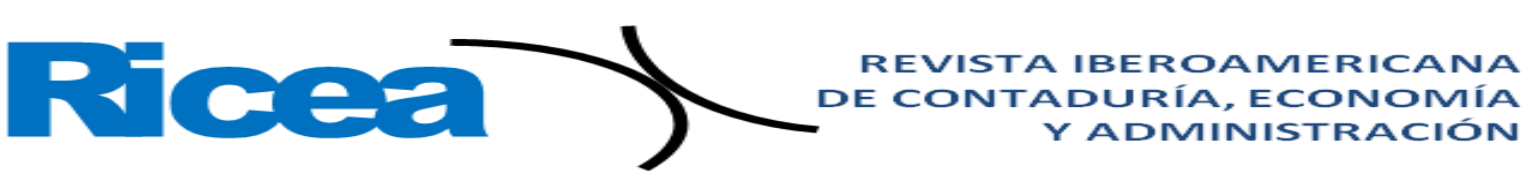

ISSN = $2007-9907$

En la figura 3 también se muestra que la dimensión "Control del proceso" tiene un valor medio de respuesta de 3.4505, lo cual indica que las mipynes en Aguascalientes tienen sistemas de control de procesos básicos y de uso general. Estos son aplicados regularmente. Por su parte, en la tabla 4 se menciona que $41.2 \%$ de las empresas utiliza solamente técnicas tradicionales de control de producción, en tanto que $58.8 \%$ utiliza frecuentemente la mayoría de las técnicas actualizadas que se relacionan con el control de los procesos.

Tabla 4. Resumen de porcentajes sencillos y acumulados de la frecuencia de respuesta para el constructo SP y sus dimensiones

\begin{tabular}{|c|c|c|c|c|c|c|c|c|}
\hline $\begin{array}{l}\text { Variables para } \\
\text { calificar el } \\
\text { constructo calidad }\end{array}$ & $\begin{array}{l}\text { Respuesta } \\
\text { principal }\end{array}$ & 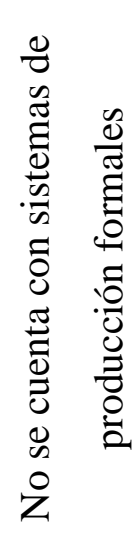 & 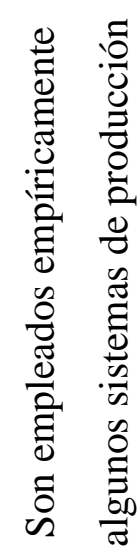 & 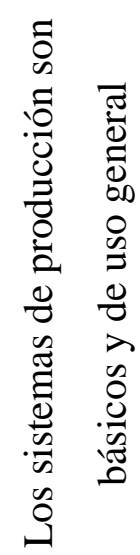 & 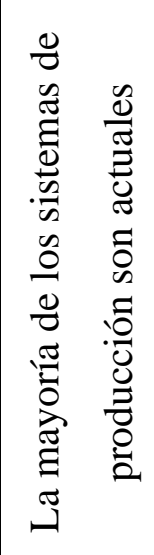 & 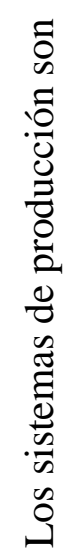 & 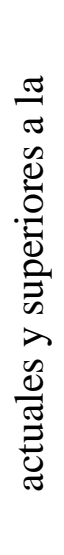 & 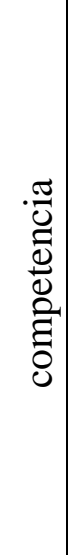 \\
\hline \multirow{3}{*}{$\begin{array}{l}\text { Automatización } \\
\text { procesos }\end{array}$} & Frecuencia & 105 & 30 & 8 & 3 & 2 & & \\
\hline & Porcentaje & 70.9 & 20.3 & 5.4 & 2 & 1.4 & & \\
\hline & Acumulado & 70.9 & 91.2 & 96.6 & 98.6 & 100 & & \\
\hline \multirow{3}{*}{ Control del proceso } & Frecuencia & 28 & 11 & 24 & 33 & 54 & & \\
\hline & Porcentaje & 17.6 & 7.4 & 16.2 & 22.3 & 36.5 & & \\
\hline & Acumulado & 17.6 & 25.0 & 41.2 & 63.5 & 100 & & \\
\hline \multirow{3}{*}{$\begin{array}{l}\text { Control } \\
\text { administrativo }\end{array}$} & Frecuencia & 42 & 29 & 48 & 17 & 12 & & \\
\hline & Porcentaje & 28.4 & 19.6 & 32.4 & 11.5 & 9.1 & & \\
\hline & Acumulado & 28.4 & 48.0 & 80.4 & 91.9 & 100 & & \\
\hline \multirow{3}{*}{$\begin{array}{l}\text { Sistemas de } \\
\text { producción }\end{array}$} & Frecuencia & 31 & 34 & 64 & 17 & 2 & & \\
\hline & Porcentaje & 20.9 & 23.0 & 43.3 & 9.4 & 1.4 & & \\
\hline & Acumulado & 20.9 & 43.9 & 87.2 & 98.6 & 100 & & \\
\hline
\end{tabular}

Fuente: Elaboración propia 


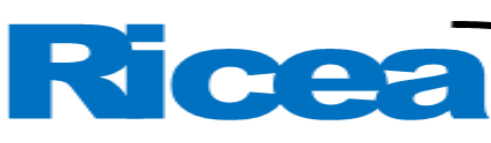

REVISTA IBEROAMERICANA DE CONTADURÍA, ECONOMIIA YADMINISTRACIÓN

ISSN= $2007-9907$

Por último, para la dimensión "Control administrativo", la figura 3 deja ver una muestra una respuesta media de 2.6, lo que indica que para esta dimensión se tienen sistemas empíricos de control y que no son empleados regularmente. Coincidentemente, la tabla 4 muestra que $80.4 \%$ de las empresas utiliza esporádicamente sistemas de control administrativos y que, en cambio, solo $19.6 \%$ se preocupa por contar sistemas de control administrativo actualizados, así como de su uso frecuente y constante.

Para determinar la relación que tienen los constructos SP y LE se realizó un análisis de correlación con las respuestas medias por empresa para cada uno de ellos. El resultado se muestra en la tabla 5. Ahí destaca que el valor de este índice es 0.574 a $99 \%$ de confiabilidad, lo que demuestra que la correlación es positiva y significativa, por lo que al mejorar el constructo SP se mejorará el de LE. Por otro lado, al calcular el valor de la $R$ cuadrada en esta correlación, se tiene un valor de 0.3294 , lo que indica que en $32.94 \%$ de los casos el estado en que se encuentra el constructo LE en las empresas se debe precisamente al estado en que se mantiene el constructo SP.

Tabla 5. Índice de correlación de los sistemas de producción y logística empresarial

\begin{tabular}{|l|l|}
\hline Correlación de Pearson & $0.574^{* *}$ \\
\hline Sig. (bilateral) & 0.000 \\
\hline **La correlación es significativa en el nivel 0.01 (bilateral). \\
\hline
\end{tabular}

Fuente: Elaboración propia

Figura 4. Diagrama de correlación SP contra LE

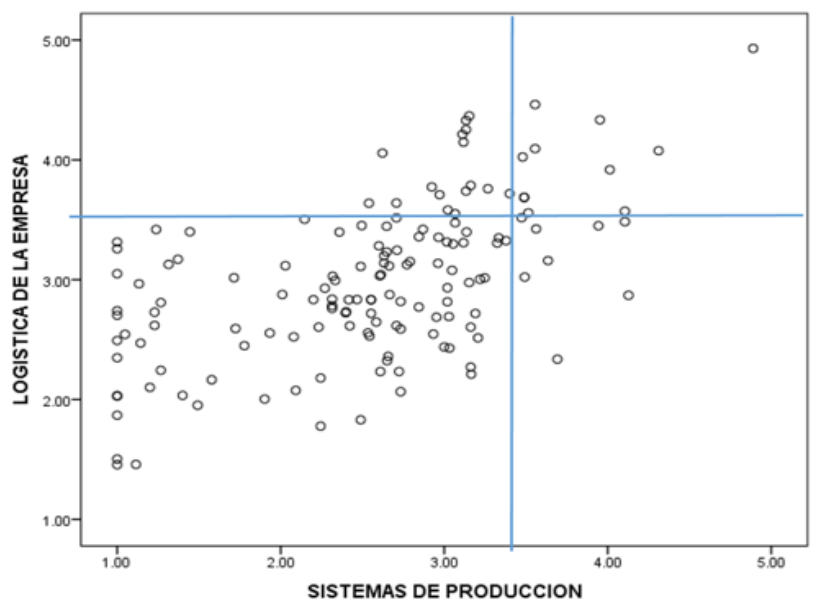

Fuente: Elaboración propia 


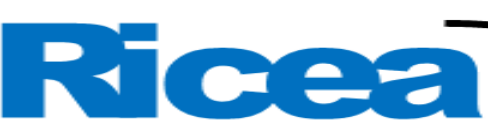

REVISTA IBEROAMERICANA DE CONTADURÍA, ECONOMÍA YADMINISTRACIÓN

ISSN : $2007-9907$

En la figura 4 se visualiza que $77.71 \%$ de los empresarios menciona que la logística empresarial en sus lugares de trabajo se encuentra en estado básico porque sus sistemas de producción se encuentran también en estado básico. Asimismo, $6.08 \%$ de los empresarios menciona que la logística en sus organizaciones se encuentra en estado de control superior, aunque sus procesos de producción se encuentren en estado básico de control. Aún más, $10.13 \%$ de los empresarios menciona que en sus organizaciones el estado del constructo LE se encuentra en niveles altos de control porque también los niveles del constructo SP se conservan altos. Por último, se puede ver también que $6.08 \%$ expresa que en sus organizaciones la logística empresarial se encuentra en niveles superiores de control a pesar de que los sistemas de producción están en niveles bajos.

Tabla 6. Resultado de la regresión simple de SP con LE

\begin{tabular}{|l|l|l|l|l|l|}
\hline & \multicolumn{2}{|l|}{$\begin{array}{l}\text { Coeficientes no } \\
\text { estandarizados }\end{array}$} & $\begin{array}{l}\text { Coeficientes } \\
\text { estandarizados }\end{array}$ & $\mathrm{t}$ & Sig. \\
\cline { 2 - 6 } & B & $\begin{array}{l}\text { Error } \\
\text { estándar }\end{array}$ & Beta & 13.49 & 0.000 \\
\hline (Constante) & 1.877 & 0.139 & & 5 & 8.473 \\
\hline SISPRO & 0.438 & 0.052 & 0.574 & 0.000 \\
\hline
\end{tabular}

Fuente: Elaboración propia

Para complementar el análisis todavía más se realizó una regresión lineal en donde se consideró al constructo SP como variable independiente y al constructo LE como dependiente. El resultado se expone en la tabla 6. De esta información, se deriva la siguiente ecuación que explica la relación entre SP y LE:

$$
\mathrm{LE}=1.877+0.438 \mathrm{SP}
$$

Comentario al margen: de las dimensiones que se utilizaron para determinar los valores de SP la de "Control administrativo" es la única que influye directamente en el comportamiento de la LE; las otras dos dimensiones no afectan con fortaleza en la manera de llevar la logística empresarial en las mipymes. 


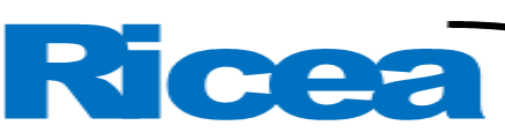

REVISTA IBEROAMERICANA DE CONTADURÍA, ECONOMÍA YADMINISTRACIÓN

\section{Discusión}

La clave para integrarse a la cadena productiva internacional es la administración de la logística de la empresa. La logística de la empresa tiene el propósito de satisfacer las demandas del cliente en el momento solicitado y con la calidad y los costos más competitivos del mercado. Hemos visto hasta aquí que el papel de los sistemas de producción es fundamental y que es indispensable que estos se modernicen técnicamente para apoyar de igual forma a la logística de la empresa. En este aspecto, es necesario que el profesionista recién integrado a la cadena productiva cuente con las credenciales suficientes para comprobar que posee el conocimiento para esta tarea y que podrá aportar técnicamente a la administración confiable de la cadena de valor de cualquier tipo de empresa.

Contrariamente, las escuelas de nivel superior están apostando a que las empresas complementen la formación del personal en el campo de trabajo. Esta situación a la empresa le cuesta hasta dos años de formación, a pesar de que el nuevo profesionista debería tener ya la capacidad para integrarse de inmediato al mercado laboral. Posiblemente esta falta de armonía entre escuela y empresa sea causada por la masificación de la educación, tal parece que se estuviera preparando mano de obra calificada para las empresas internacionales y no para soportar y desarrollar la empresa local.

Uno de los principales problemas en los sistemas de producción de las empresas locales es la falta de automatización de los procesos, lo cual, a su vez, es motivado por el retraso tecnológico de nuestro país y la falta de financiamiento accesible para motivar a la empresa a mejorar en este aspecto. La empresa, para paliar esta deficiencia, debe aplicar con mayor fuerza herramientas de análisis y mejora de operaciones, para el desarrollo de ayudas de trabajo, así como a considerar llevar a cabo estrategias de modernización de los equipos y la maquinaria aplicando los apoyos financieros como es la depreciación de activos, lo cual, si se llevara a cabo como corresponde, daría la oportunidad de contar con equipo y maquinaria actualizados.

El trabajo para que las mipymes logren desarrollarse es muy arduo, implica un compromiso del propietario o administrador principal. Debemos tener presente siempre que las empresas grandes, en alguna ocasión, también fueron mipymes y que fue gracias al apoyo de las políticas públicas y el esfuerzo propio que lograron el desarrollo que se puede observar actualmente. 


\section{Conclusiones}

El estudio revela que las mipymes muestran un atraso sustancial en la aplicación de herramientas direccionadas a optimizar la administración de los procesos que forman parte del trabajo que se realiza en ellas. La brecha técnica entre estas y empresas internacionales es cada vez mayor, por lo que el dominio en el mercado es patente. Tanto las políticas públicas como la administración de este tipo de organizaciones han dado pauta a que esta brecha sea cada vez mayor, y como consecuencia, se está a la disposición de lo que se haga en las empresas internacionales, por lo tanto, el dominio de país a país de igual manera se manifiesta.

Se debe tener en claro que los resultados de esta investigación muestran que los sistemas de producción tienen un retraso considerable respecto a las empresas que llegan nuestro país del extranjero, y que en muchos casos se tienen equipos y máquinas primitivas, por lo que no se puede competir con ellas, y por lo que cualquier tratado que se realice con potencias mundiales siempre será desfavorable para las empresas locales. La preocupación por el progreso se debe direccionar primeramente a fortalecer a la industria local para poder establecer convenios con países desarrollados en igualdad de circunstancias.

\section{Futuras líneas de Investigación}

Se propone que para futuras líneas de investigación se considere ampliar la muestra a empresas de tamaño grande, además de que se puede replicar este estudio en diferentes áreas geográficas del país para poder comparar los resultados. 


\section{Referencias}

Ahmad, K. A., Tahir, M., Ul, I. and Shah, K. (2019). Agent-based fault tolerant framework for manufacturing process automation. International Journal of Computer Integrated Manufacturing 32(3), 268-277.

Bay, C. (2021). El G7 de las grandes potencias: ¿desde cuándo se celebra y qué países han formado parte de este 'club de ricos'? El Mundo. Recuperado de https://www.elmundo.es/internacional/2021/06/11/60c3554afc6c83141a8b4590.htm 1.

Boulter, L., Bendell, T. and Dahlgaard, J. J. (2013). Total quality beyond North America: A comparative analysis of the performance of European Excellence Award winners. International Journal of Operations \& Production Management, 33(2), 311-320.

Bowersox, D. J., Closs, D. J., Cooper, M. B. e Bowersox J. C. (2013). Gestão logística da cadeia de suprimentos. Brasil: AMGH Editora

Dahlgaard, S. M. (2011). The quality movement: Where are you going? Total Quality Management \& Business Excellence, 22(5), 493-516.

Gershwin, S. B. and Schor, J. E. (2000). Efficient algorithms for buffer space allocation. Annals of Operations Research, 93(1), 117-144.

Gola, A. and Konczal, W. (2013) RMS - system of the future or new trend in science? Advances in Science and Technology, 7(20), 35-41. Retrieved from http://dx.doi.org/10.5604/20804075.1073052.

Gustaffson, A. (2006). Customers' logistics service requirements and logistics strategies in the Swedish sawmill industry. (Doctoral thesis). Vaxjo University, Sweden.

Hu, W., Starr, A. G. y Leung, A. Y. T. (1999). Two diagnostic models for PLC controlled flexible manufacturing systems. International Journal of Machine Tools and manufacture. 39 (12): 1979 -1999. Doi: 10.1016/S08090-6955(99)00022-X

Hymer, S. H. (1976). The International Operations of National Firms: A Study of Direct Foreign Investment. Cambridge, United States: MIT Press.

Instituto Nacional de Estadística y Geografía (INEGI). (2009). Micro, pequeña, mediana y gran empresa. Recuperado de https://www.inegi.org.mx/contenidos/programas/ce/2009/doc/minimonografias/m_p ymes.pdf. 
Lee, H. F. (1998). Production planning for flexible manufacturing systems with multiple machine types: a practical method. International Journal Production Research, $36(10), 2911-2927$

Jellouli, O. and Adbelkadhi, M. (2013). Tests Logistics Maturity of the Industrial Zone in the Region of Gabes. International Journal of Supply Chain Management, 2(4), 20502073.

Lovitt, M. R. (1997). The new pragmatism: Going beyond Shewhart and Deming. Quality Progress, 30(4), 99-105.

Milberg, W. (2004). Cambio del comercio ligado a los sistemas mundiales de producción. ¿Qué política seguir? Revista Internacional del Trabajo, 123(1, 2), 49-101.

Noori, H. y Radford, R. (1997). Administración de operaciones y producción. Santa Fe, Colombia: McGraw-Hill.

Nunnally, J. C. and Bernstein, I. H. (1994). Psychometric Theory ( $3^{\text {rd }}$ ed.). New York, United States: McGraw-Hill.

Pascual, R. C. and Ribas, I. (2015). Some trends and applications of operational research/management science to operations management. International Journal of Production Management and Engineering, 3(1), 1-12. Retrieved from http://dx.doi.org/10.4995/ijpme.2015.3459.

Peters, T. (1987). Thriving on Chaos: Handbook for a Management Revolution. New York, United States: Alfred A. Knopf.

Ranta, J. and Tchijov, I. (1990). Economics and Success Factors of Flexible Manufacturing Systems: The Conventional Explanation Revisited. International Journal of Flexible Manufacturing Systems, 2(3), 169-190.

Ruzzier, M., Hisrich, R. D. and Antoncic, B. (2006). SME internationalization research: past, present and future. Journal of Small Business and Enterprise Development, 13(4), 476-497.

Samaranayake, P. and Toncich, D. (2007). Integration of production planning, project management and logistics systems for supply chain management. International Journal of Production Research, 45(22), 5417-5447.

Sandberg, E. (2013). Understanding logistics-based competition in retail - a business model approach. International Journal of Retail \& Distribution Management, 41(1), 176188. Retrieved from http://dx.doi.org/10.1108/09590551311306237. 
Smith, T. and Stecke, K. E. (1996). On robustness of using balanced part mix ratios to determine cyclic part input sequences into flexible flow systems. International Journal of Production Research, 34(10), 2925-2941.

\begin{tabular}{|c|c|}
\hline Rol de Contribución & Autor (es) \\
\hline Conceptualización & Salomón Montejano García \\
\hline Metodología & Salomón Montejano García \\
\hline Software & No aplica \\
\hline Validación & Gabriela Citlalli López Torres \\
\hline Análisis Formal & Marcelo de Jesús Pérez Ramos \\
\hline Investigación & Marcelo de Jesús Pérez Ramos \\
\hline Recursos & Rocío Montserrat Campos García \\
\hline Curación de datos & Marcelo de Jesús Pérez Ramos \\
\hline $\begin{array}{l}\text { Escritura - Preparación del } \\
\text { borrador original }\end{array}$ & Salomón Montejano García \\
\hline $\begin{array}{l}\text { Escritura - Revisión y } \\
\text { edición }\end{array}$ & Rocío Montserrat Campos García \\
\hline Visualización & Gabriela Citlalli López Torres \\
\hline Supervisión & Salomón Montejano García \\
\hline Administración de Proyectos & Salomón Montejano García \\
\hline Adquisición de fondos & Rocío Montserrat Campos García \\
\hline
\end{tabular}

